\title{
Validation of the SenseWear armband in circuit resistance training with different loads
}

\author{
P. J. Benito - C. Neiva · P. S. González-Quijano • \\ R. Cupeiro $\cdot$ E. Morencos $\cdot$ A. B. Peinado
}

\begin{abstract}
The use of the SenseWear ${ }^{\mathrm{TM}}$ armband (SWA), an objective monitor of physical activity, is a relatively new device used by researchers to measure energy expenditure. These monitors are practical, relatively inexpensive and easy-to-use. The aim of the present study was to assess the validity of SWAs for the measurement of energy expenditure (EE) in circuit resistance training (CRT) at three different intensities in moderately active, healthy subjects. The study subjects ( 17 females, 12 males) undertook CRT at 30,50 and $70 \%$ of the 15 repetition maximum for each exercise component wearing an SWA as well as an Oxycon Mobile (OM) portable metabolic system (a gold standard method for measuring EE). The EE rose as exercise intensity increased, but was underestimated by the SWAs. For women, Bland-Altman plots showed a bias of $1.13 \pm 1.48$ METs and $32.1 \pm 34.0 \mathrm{kcal}$ in favour of the OM system, while for men values of $2.33 \pm 1.82$ METs and $75.8 \pm 50.8 \mathrm{kcal}$ were recorded.
\end{abstract}

Communicated by Klaas R Westerterp.

P. J. Benito $(\triangle)$ · P. S. González-Quijano - R. Cupeiro ·

E. Morencos · A. B. Peinado

Department of Health and Human Performance,

Faculty of Physical Activity and Sport Sciences,

Universidad Politécnica de Madrid, C/Martín Fierro 7,

28040 Madrid, Spain

e-mail: pedroj.benito@upm.es

C. Neiva

Laboratory of Metabolism and Exercise Physiology (MEFE),

Faculty of Science, Universidade Estadual Paulista-UNESP,

São Paulo, Brazil

C. Neiva

Graduate Program in Health Promotion, Universidade de

Franca-UNIFRAN, São Paulo, Brazil
Keywords Physical activity assessment - Energy expenditure $\cdot$ Accelerometer $\cdot$ Resistance training

\section{Introduction}

Resistance exercise is recommended by the American College of Sports Medicine (ACSM), the American Heart Association (AHA) and the National Strength and Conditioning Association (NSCA). Among its benefits are increased muscular strength and endurance, and reduced blood pressure (Kelley and Kelley 2000). In addition, since resistance exercise raises energy expenditure (EE), it could help prevent weight gain after its loss (Hunter et al. 2008). It could, therefore, be of value during weight loss or weight loss maintenance programs when combined with energy intake restriction and endurance exercise (Kraemer et al. 1999).

The use of systems for measuring EE during resistance training is of great importance, especially when the main purpose is to lose or control body weight (Trost 2001). Several authors have attempted to validate the methods, techniques and equipment for the analysis of EE using different populations taking part in different forms of physical training (Rawson and Walsh 2010; Schoeller 1988; Trost 2001). Some have tried to determine the EE during strength training using gold standard assessment systems such as the doubly labelled water (DLW) technique (Schoeller 1988), respiratory gas analysis and the measurement of biochemical markers in blood (St-Onge et al. 2007). However, these methods are either laborious or expensive. SenseWear ${ }^{\mathrm{TM}}$ Pro2 armband (SWA) accelerometers were designed to offer a cheap, easy-to-use alternative, but they are reported to underestimate EE during high intensity exercise (Drenowatz and Eisenmann 2011). 
The accuracy of SWAs in resistance and interval training with different workloads has not been tested.

The aim of the present study was to compare the results for EE provided by SWAs and by respiratory gas analysis using an Oxycon Mobile (OM) portable metabolic system (Erich Jaeger, Viasys Healthcare, Germany) during circuit resistance training (CRT) under different loads in young men and women.

\section{Methods}

The study subjects were 12 men (age $24.2 \pm 1.9$ years; weight $79.5 \pm 7.2 \mathrm{~kg}$; height $180.6 \pm 6.8 \mathrm{~cm}$ ) and 17 women (age $21.5 \pm 2.6$ years; weight $58.8 \pm 4.9 \mathrm{~kg}$; height $162.7 \pm 3.7 \mathrm{~cm}$ ). All were moderately active (undertaking $3-5 \mathrm{~h}$ exercise week ${ }^{-1}$ and with at least 1 year of experience in strength training), as assessed using the criteria of the International Physical Activity Questionnaire (IPAQ) (short version). None of the subjects were smokers, had diabetes, cardiovascular disease or metabolic disorders (potential subjects with these conditions were excluded). The medical histories of the subjects were checked and physical examinations performed to confirm suitability for inclusion. All subjects gave their written informed consent to participate in the study, which was approved by the Human Research Review Ethic Committee of the Universidad Politécnica de Madrid.

Each subject came to the laboratory eight times. During the first visit, the resting metabolic rate (RMR) was obtained. The next four visits involved the calculation of the 15 repetition maximum (15 RM) from each of the different components of the CRT. In the final three visits, CRT was performed at three different intensities (randomised counterbalance order): 30,50 and $70 \%$ of $15 \mathrm{RM}$ for each CRT component.

To assess the RMR, participants arrived at the laboratory at 7:00 a.m. after a $12 \mathrm{~h}$ overnight fast. The RMR was measured by indirect calorimetry in the supine position (over $30 \mathrm{~min}$ ) and standing up (over an additional $15 \mathrm{~min}$ ), according to Melanson et al. (2002).

The 15 RM for each component exercise was determined over 2 weeks with a maximum of two attempts per component per day. The test started after a 5-min cardiovascular warm-up on a cycle ergometer, followed by three sets of 15 repetitions of the exercise component for the day performed at 50, 70 and $90 \%$ of the estimated 15 RM. A 2 -min recovery period was allowed between attempts. The subjects then rested for $5 \mathrm{~min}$ before undertaking a final set of 15 repetitions at $100 \%$ of the estimated 15 RM. If the subject was able to exceed 15 repetitions, a further attempt was performed after 5 min recovery, at $+2.5 \%$ of the estimated $15 \mathrm{RM}$. If the subject was unable to manage 15 repetitions, the load was reduced by $2.5 \%$ (Hurley et al. 1988; Scott and Kemp 2005). All tests were performed with the same cadence $(2: 1)$ to be used later during the CRT protocols. During tests, all the subjects were encouraged to perform as many repetitions as possible.

Prior to starting a CRT session, an SWA was placed on the upper dominant arm (on the triceps) of each volunteer. Breath-by-breath analysis of the expired gases was undertaken over the entire session using an OM (Erich Jaeger, Viasys Healthcare) portable metabolic system (Díaz et al. 2008). The OM system was calibrated prior to each subject-session; oxygen consumption was recorded in $10 \mathrm{~s}$ intervals. Heart rate (HR) was recorded using an $\mathrm{HR}$ monitor (Polar Electro Oy, Kempele, Finland) functioning alongside the gas analyser. Each session started by performing a warm-up involving 5 min running on a treadmill at $50 \%$ of the heart rate reserve, followed by $1 \mathrm{~min}$ rest and then a CRT lap at just $20 \%$ of 15 RM. A 1 -min recovery was then allowed before the subjects performed three CRT laps at either 30,50 or $70 \%$ of the $15 \mathrm{RM}$ for each component. All the exercises were performed on Pannata exercise machines (Pannata, Italy) and in the following order: (1) sitting bench press, (2) leg extension, (3) lat pulldown, (4) inclined leg press, (5) shoulder press, (6) leg curl, (7) biceps curl and (8) cable push downs.

Exercise EE for aerobic metabolism was converted as $1 \mathrm{~L}$ of $\mathrm{O}_{2}=5 \mathrm{kcal}$. Upon completion of the session, subjects immediately rested standing up, and the excess post-exercise oxygen consumption (EPOC) was recorded using the OM until it fell below the respective 10 min resting $\mathrm{O}_{2}$ uptake recorded in the RMR test. The EPOC was calculated as $1 \mathrm{~L}$ of $\mathrm{O}_{2}$ consumed $=4.64 \mathrm{kcal}$ used ( $\mathrm{Scott}$ and Kemp 2005). The latter figure was assumed to exclude rapid glycolytic ATP re-synthesis as part of EE; thus EPOC represented aerobic EE only (Scott and Kemp 2005).

The portable SWAs (HealthWear, Bodymedia, Pittsburgh, PA, USA) used in this work were composed of a two-axis accelerometer, a heat flux sensor, a galvanic skin response sensor, a skin temperature sensor and a near-body ambient temperature sensor. The data they recorded, as well as gender, body weight, height, handedness and smoking status (smoker or non-smoker), were used to calculate EE based on the SWA proprietary algorithm. Second-by-second values are reported as either kcal or METs. Data were downloaded to a computer using INNERVIEW version 4.02 software (Bodymedia).

\section{Statistical analyses}

The normal distribution of the data was confirmed using the Kolmogorov-Smirnov test. Differences in mean SWAand OM-determined EE values were examined using the paired Student $t$-test. Bland-Altman plots were drawn to 
establish the bias of the EE values for one measuring system or the other following the prescribed procedure (Bland and Altman 1986). Three-way ANOVA (gender $\times$ devices $x$ intensities) with repeated measures was used to analyse differences between EE values (in kcal and METs). This was followed by two-way ANOVA (gender $\times$ intensities) with repeated measures. Multiple comparisons of EE between sexes and intensities were performed using the Bonferroni post hoc test. All analyses were performed using SPSS 15.0 software for Windows (SPSS, Chicago, IL, USA). Significance was set at $p<0.05$.

\section{Results}

Significant differences were detected in the EE results (both in kcal and in METs) provided by the two devices at all exercise intensities (Table 1). These differences persisted when all intensity results were grouped and analysed together (Table 1).

In general, the SWA system underestimated the EE (Fig. 1). For the women, Bland-Altman plots showed a bias of $1.13 \pm 1.48$ METs and $32.1 \pm 34.0 \mathrm{kcal}$ in favour of the OM system, while for men values of $2.33 \pm 1.82$ METs and $75.8 \pm 50.8 \mathrm{kcal}$ were recorded.

Figure 2 reveals significant differences between the delta values (the difference in $\mathrm{EE}$ as measured by $\mathrm{OM}$ and SWA) of the men and women at the 50 and $70 \%$ intensities, but not at the $30 \%$ intensity. No differences were seen, however, at any intensity among the women. Thus, the difference between the devices becomes greater as exercise increases, but only in men. Three-way ANOVA confirmed the interaction intensity $\times$ sex to have a significant effect on the difference in EE values measured by the two systems (in kcal $F[2.1]=5.645, p=0.016$; in METs, $F[2.1]=4.197, p=0.036$ ) (Fig. 2).

\section{Discussion}

Some authors (St-Onge et al. 2007) report a strong correlation between estimates of EE made by SWAs and indirect calorimetry during low intensity exercise. Further, in comparisons with indirect calorimetric methods and the DLW test, Drenowatz and Eisenmann (2011) reported SWAs to accurately determine EE in adults and children at rest and during low to moderate intensity exercise. However, the present results show that SWAs underestimate EE during CRT, with the error increasing as exercise intensity rises in men. Drenowatz and Eisenmann (2011) also reported SWAs to inaccurately estimate $\mathrm{EE}$ at intensities above 10 METs or at a running speed of above $6 \mathrm{mph}$ $\left(161 \mathrm{~m} \mathrm{~min}^{-1}\right)$. While the three exercise intensities they investigated returned similar values for $\mathrm{EE}(9.36,9.60$ and 9.51 METs for 65,75 and $85 \%$ of $\dot{V} \mathrm{O}_{2 \max }$, respectively) when measured by SWAs, OM-based measurements showed EE to increase with exercise intensity. They suggested the cause might lie in the algorithm used by the apparatus. Koehler et al. (2010) also reported inaccuracies when studying men running for $5 \mathrm{~min}$ at speeds above $6.3 \mathrm{mph}$. The results of both latter studies suggest that above 10 METS, the results provided by SWAs become very unreliable.

These problems may be explained in that the SWA algorithm relies on the measurement of body acceleration;
Table 1 Mean $( \pm S D)$ values for SWA and OM estimations at different intensities
Values are mean $( \pm$ SD)

$\dagger$ Significant differences between devices SWA vs. OM $(p<0.01)$

\begin{tabular}{llrrr}
\hline Variable & $\begin{array}{l}\text { Intensity } \\
(\%)\end{array}$ & \multicolumn{1}{l}{ Males } & \multicolumn{1}{l}{ Females } & \multicolumn{1}{l}{ Total } \\
\hline OM energy expenditure & 30 & $5.45( \pm 1.07)$ & $4.49( \pm 1.36)$ & $4.89( \pm 1.32)$ \\
(METs) & 50 & $7.52( \pm 1.15)$ & $5.27( \pm 1.37)$ & $6.10( \pm 1.68)$ \\
& 70 & $9.39( \pm 2.32)$ & $5.96( \pm 1.51)$ & $7.24( \pm 2.47)$ \\
& All & $7.26( \pm 2.25)$ & $5.33( \pm 1.58)$ & $6.06( \pm 2.08)$ \\
SWA energy expenditure & 30 & $4.42^{\dagger}( \pm 0.69)$ & $3.83^{\dagger}( \pm 0.64)$ & $4.07^{\dagger}( \pm 0.72)$ \\
(METs) & 50 & $4.87^{\dagger}( \pm 0.88)$ & $4.14^{\dagger}( \pm 0.61)$ & $4.41^{\dagger}( \pm 0.79)$ \\
& 70 & $5.68^{\dagger}( \pm 0.92)$ & $4.74^{\dagger}( \pm 0.54)$ & $5.09^{\dagger}( \pm 0.83)$ \\
& All & $4.93^{\dagger}( \pm 1.05)$ & $4.20^{\dagger}( \pm 0.68)$ & $4.48^{\dagger}( \pm 0.91)$ \\
OM energy expenditure & 30 & $157.81( \pm 26.67)$ & $97.72( \pm 30.75)$ & $122.58( \pm 41.56)$ \\
(Kcal) & 50 & $218.34( \pm 36.83)$ & $114.10( \pm 28.54)$ & $152.71( \pm 60.02)$ \\
& 70 & $266.09( \pm 52.74)$ & $129.64( \pm 32.37)$ & $180.18( \pm 78.21)$ \\
& All & $208.13( \pm 60.39)$ & $116.36( \pm 36.12)$ & $151.03( \pm 64.52)$ \\
SWA energy expenditure & 30 & $116.58^{\dagger}( \pm 16.60)$ & $74.59^{\dagger}( \pm 8.64)$ & $91.97^{\dagger}( \pm 24.37)$ \\
(Kcal) & 50 & $135.20^{\dagger}( \pm 15.61)$ & $82.94^{\dagger}( \pm 12.39)$ & $102.30^{\dagger}( \pm 28.99)$ \\
& 70 & $148.80^{\dagger}( \pm 13.39)$ & $97.35^{\dagger}( \pm 12.67)$ & $116.41^{\dagger}( \pm 28.32)$ \\
& All & $132.33^{\dagger}( \pm 19.74)$ & $84.25^{\dagger}( \pm 13.74)$ & $102.41^{\dagger}( \pm 28.46)$ \\
\hline
\end{tabular}



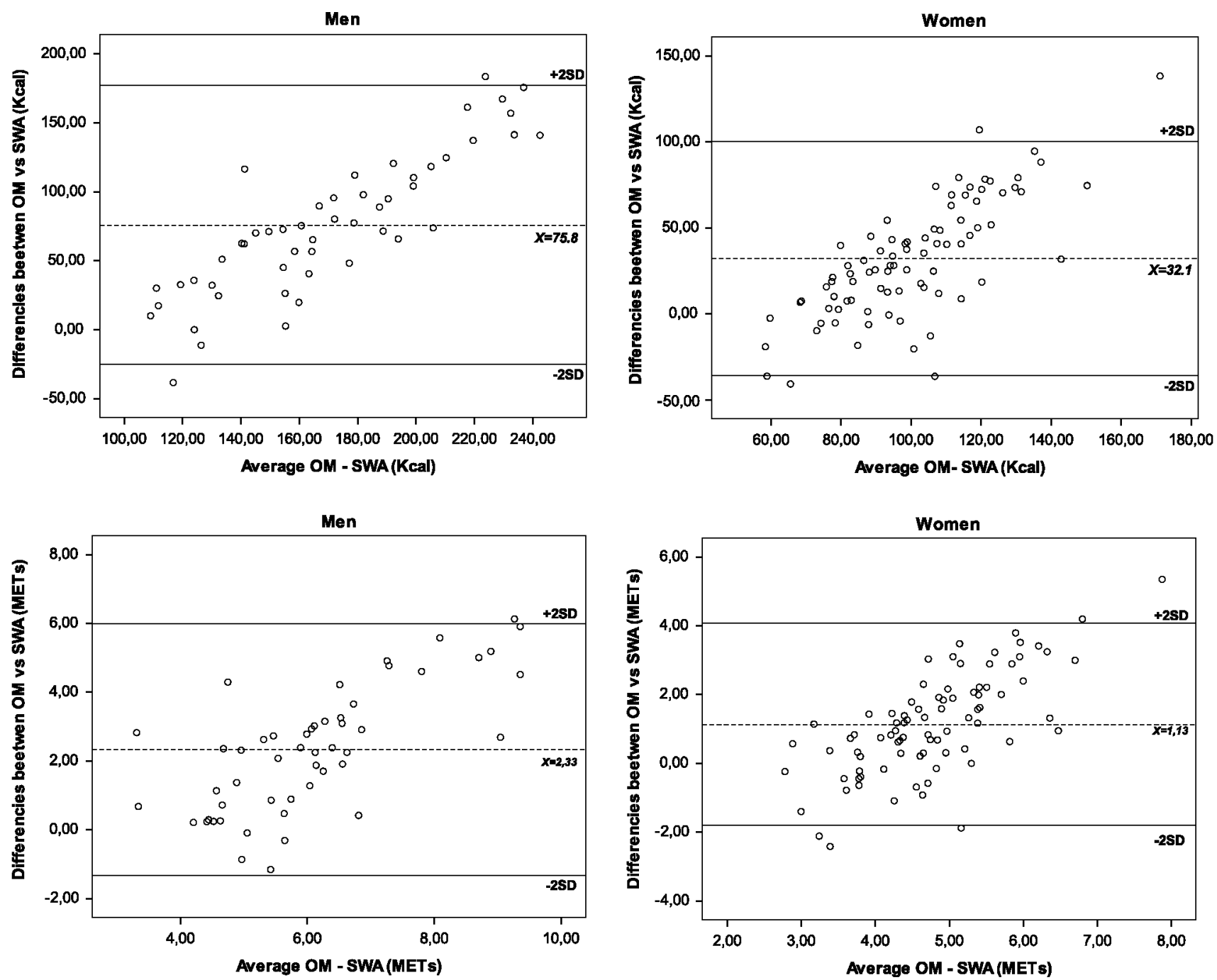

Fig. 1 Bland-Altman plots for OM/SWA for both men and women (data expressed in METs and Kcal)

however, it does not take into account the actual load being moved. Thus, the EE results provided by the algorithm would be the same whether 20 or $50 \mathrm{~kg}$ were being moved if the acceleration of the body moving them were the same. Any underestimation of EE might, therefore, become greater, especially in men, as exercise intensity increases.

The underestimation of EE in men compared to women with increasing exercise intensity may be explained in that women may perceive their effort as being greater than it truly is. Thus, the present $15 \mathrm{RM}$ for the women may not have truly reflected their maximum effort. In studies of resistance exercise training for the management of body weight, Benton and Swan (2009) concluded that women should be encouraged to exercise at higher intensities than they believe to be their best effort (e.g. 70-80\% 1 RM) in order to maximise the volume of work and the energy expended.

Drenowatz and Eisenmann (2011) suggest that, despite taking into account heat-related measurements, SWAs might fail to overcome their limitations at higher exercise intensities since sweating rates increase with exercise intensity. This might lead to sensors returning inaccurate readings. The site where an accelerometer is worn may also influence the EE results obtained (Johannsen et al. 2008), although in the present work the SWAs were adequately worn on the triceps.

It is possible that the SWA propriety algorithms make use of regression equations to calculate EE. However, if they are based on those for walking, jogging and the activities of daily living, EE during CRT exercise may not be accurately measured. Similar concerns were expressed by Rawson and Walsh (2010) in a study involving accelerometers made by a different manufacturer.

As suggested by Koehler et al. (2010) and Drenowatz and Eisenmann (2011), the present results suggest that the SWA algorithm may need to be modified for use with resistance training. The addition of heart rate measurements (Plasqui and Westerterp 2005) or better body 

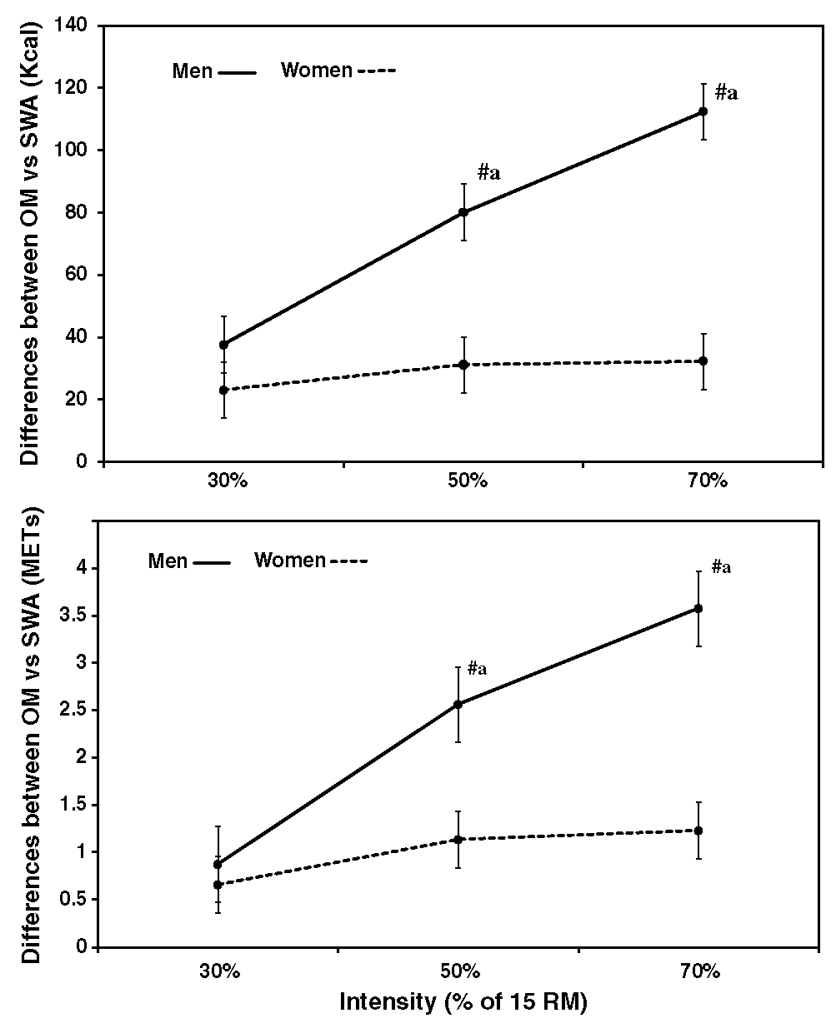

Fig. 2 Delta differences for OM/SWA at each intensity for men and women (in METs and Kcal). Values are shown as means and standard errors. ${ }^{a}$ Significantly different compared to $30 \%$ of $15 \mathrm{RM}$ $(p<0.001)$. "Significantly different between sexes $(p<0.001)$

tracking (Rawson and Walsh 2010), perhaps achieved through the use of triaxial accelerometers, may also be useful. Further work using SWAs that include these modifications should be undertaken.

\section{References}

Benton MJ, Swan PD (2009) Influence of resistance exercise volume on recovery energy expenditure in women. Eur $\mathbf{J}$ Sport Sci $4(9): 213-218$

Bland JM, Altman DG (1986) Statistical methods for assessing agreement between two methods of clinical measurement. Lancet 1(8476):307-310
Díaz V, Benito PJ, Peinado AB, Álvarez M, Martín C, di Salvo V, Pigozzi F, Maffulli N, Calderón FJ (2008) Validation of a new portable metabolic system during an incremental running test. J Sport Sci Med 7(4):532-536

Drenowatz C, Eisenmann JC (2011) Validation of the SenseWear Armband at high intensity exercise. Eur J Appl Physiol 111(5):883-887

Hunter GR, Byrne NM, Sirikul B, Fernandez JR, Zuckerman PA, Darnell BE, Gower BA (2008) Resistance training conserves fatfree mass and resting energy expenditure following weight loss. Obesity (Silver Spring, MD) 16(5):1045-1051

Hurley BF, Hagberg JM, Goldberg AP, Seals DR, Ehsani AA, Brennan RE, Holloszy JO (1988) Resistive training can reduce coronary risk factors without altering VO2max or percent body fat. Med Sci Sports Exerc 20(2):150-154

Johannsen DL, Welk GJ, Sharp RL, Flakoll PJ (2008) Differences in daily energy expenditure in lean and obese women: the role of posture allocation. Obesity (Silver Spring) 16(1):34-39

Kelley GA, Kelley KS (2000) Progressive resistance exercise and resting blood pressure: a meta-analysis of randomized controlled trials. Hypertension 35(3):838-843

Koehler K, de Marees M, Braun H, Schaenzer W (2010) Comparison of two portable devices for assessing energy expenditure during high-intensity running. Med Sci Sports Exerc 42(5):433

Kraemer WJ, Volek JS, Clark KL, Gordon SE, Puhl SM, Koziris LP, McBride JM, Triplett-McBride NT, Putukian M, Newton RU, Hakkinen K, Bush JA, Sebastianelli WJ (1999) Influence of exercise training on physiological and performance changes with weight loss in men. Med Sci Sports Exerc 31(9): 1320-1329

Melanson EL, Sharp TA, Seagle HM, Donahoo WT, Grunwald GK, Peters JC, Hamilton JT, Hill JO (2002) Resistance and aerobic exercise have similar effects on 24-h nutrient oxidation. Med Sci Sports Exere 34(11): 1793-1800

Plasqui G, Westerterp KR (2005) Accelerometry and heart rate as a measure of physical fitness: proof of concept. Med Sci Sports Exerc 37(5):872-876

Rawson ES, Walsh TM (2010) Estimation of resistance exercise energy expenditure using accelerometry. Med Sci Sports Exerc 42(3):622-628

Schoeller DA (1988) Measurement of energy expenditure in freeliving humans by using doubly labeled water. J Nutr 118(11):1278-1289

Scott CB, Kemp RB (2005) Direct and indirect calorimetry of lactate oxidation: implications for whole-body energy expenditure. J Sports Sci 23(1):15-19

St-Onge M, Mignault D, Allison DB, Rabasa-Lhoret R (2007) Evaluation of a portable device to measure daily energy expenditure in free-living adults. Am J Clin Nutr 85(3):742-749

Trost SG (2001) Objective measurement of physical activity in youth: current issues, future directions. Exerc Sport Sci Rev 29(1): $32-36$ 